PROCEEDINGS OF THE

AMERICAN MATHEMATICAL SOCIETY

Volume 126, Number 6, June 1998, Pages 1673-1683

S 0002-9939(98)04291-9

\title{
FINITE RANK SINGULAR PERTURBATIONS AND DISTRIBUTIONS WITH DISCONTINUOUS TEST FUNCTIONS
}

\author{
P. KURASOV AND J. BOMAN
}

(Communicated by Palle E. T. Jorgensen)

\begin{abstract}
Point interactions for the $n$-th derivative operator in one dimension are investigated. Every such perturbed operator coincides with a selfadjoint extension of the $n$-th derivative operator restricted to the set of functions vanishing in a neighborhood of the singular point. It is proven that the selfadjoint extensions can be described by the planes in the space of boundary values which are Lagrangian with respect to the symplectic form determined by the adjoint operator. A distribution theory with discontinuous test functions is developed in order to determine the selfadjoint operator corresponding to the formal expression

$$
L=\left(i \frac{d}{d x}\right)^{n}+\sum_{l, m=0}^{n-1} c_{l m} \delta^{(m)}(\cdot) \delta^{(l)}, \quad c_{l m}=\overline{c_{m l}}
$$

representing a finite rank perturbation of the $n$-th derivative operator with the support at the origin.
\end{abstract}

\section{INTRODUCTION}

Finite rank perturbations of the $n$-th derivative operator $L_{0}=\left(i \frac{d}{d x}\right)^{n}$ in $L_{2}(\mathbf{R})$ are studied in the present paper. Consider perturbations $V$ that are bounded with respect to the original operator $L_{0}$ in the form sense, i.e.

$$
|\langle V \psi, \psi\rangle| \leq a\left\langle L_{0} \psi, \psi\right\rangle+b\langle\psi, \psi\rangle
$$

for all $\psi \in \operatorname{Dom}\left(L_{0}\right)$. Here $\langle\cdot, \cdot\rangle$ denotes the scalar product in the Hilbert space $L_{2}(\mathbf{R})$. According to the KLMN theorem ([ReSi]) a perturbation satisfying (1.1) for some constant $a<1$ determines the perturbed operator uniquely via the sum of the corresponding quadratic forms (if $L_{0}$ is positive). Our aim is to show that perturbations which do not satisfy the latter condition can be defined using in addition homogeneity properties of the original operator and the perturbation. Such operators were studied for the first time by F. Berezin and L. Faddeev [BeFad]. The book by S. Albeverio, F. Gesztesy, R. Hoegh-Krohn, and H. Holden [AGHKH] contains numerous examples. Similar problems when the perturbation has rank one have been studied recently by B. Simon, F. Gesztesy, A. Kiselev, S. Albeverio, and P. Kurasov ([Si], [KiSi], [GeSi], [AlKu], [AlKu2], [AlKu3]).

We study the finite rank perturbations of $L_{0}$ that are supported by one point, i.e. perturbations $V$ such that $\langle V \psi, \varphi\rangle=0$ when $\psi$ or $\varphi$ has support separated from

Received by the editors November 7, 1996.

1991 Mathematics Subject Classification. Primary 34L40, 46F10, 47A55, 81Q15. 
the origin. Any such perturbed selfadjoint operator coincides with some selfadjoint extension of the operator $L_{0}$ restricted to the set of functions vanishing in a neighbourhood of the origin. This operator has deficiency indices $(n, n)$, and the set of its selfadjoint extensions is described by unitary $n \times n$ matrices via von Neumann formulas. We consider the symmetric perturbation at the origin of the operator $L_{0}$ described by the formal expression

$$
L=\left(i \frac{d}{d x}\right)^{n}+\sum_{l, m=0}^{n-1} c_{l m} \delta^{(m)}(\cdot) \delta^{(l)},
$$

where the coefficients $c_{l m}$ form an $n \times n$ Hermitian matrix. The latter formal expression does not define a selfadjoint operator on the set of functions $n-1$ times continuously differentiable at the origin, but the perturbation is defined on such functions only. The quadratic form approach does not determine a selfadjoint perturbed operator for arbitrary coefficients $c_{l m}$. To define a selfadjoint operator corresponding to the formal expression (1.2) a distribution theory for discontinous test functions is developed. Such theory is considered in Section 2. We discuss the possibility of extending the definitions of the delta function and its derivatives to test functions that are discontinuous at the origin. It is proven that homogeneous extensions of those distributions exist and are unique. Then the selfadjoint operator corresponding to the formal expression (1.2) is determined in Section 3. It is shown that perturbed operators of the kind discussed here can be defined even for infinite coefficients $c_{l m}$. It is proven that the considered set of finite rank perturbations of the operator $L_{0}$ coincides with the set of selfadjoint extensions of $L_{0}$ restricted to the set of functions with support outside the origin.

\section{Distribution THEORY FOR DISCONTINUOUS TEST FUNCTIONS}

2.1. Test functions and distributions. We introduce the space $K$ of test functions as follows.

Definition 2.1. The set of test functions $K$ is the set of all functions defined on $\mathbf{R} \backslash\{0\}$ with support contained in some bounded interval and having bounded derivatives of all orders.

Thus the functions in $K$ can be discontinuous at the origin, but the limits of the functions and all derivatives from the left and from the right of the point zero exist and are finite. Convergence in this space is defined as follows.

Definition 2.2. A sequence $\left\{\varphi_{n}\right\}$ of functions in $K$ is said to converge to a function $\varphi \in K$ if and only if

1) there exists an interval outside which all the functions $\varphi_{n}$ vanish;

2) for any $k$ the sequence $\left\{\varphi_{n}^{(k)}\right\}$ of derivatives of order $k$ converges uniformly to $\varphi^{(k)}$.

Let $\mathbf{R}_{+}$denote the closed positive half-axis, and let $\mathcal{D}\left(\mathbf{R}_{+}\right)$denote the set of restrictions to $\mathbf{R}_{+}$of functions from $\mathcal{D}(\mathbf{R})=C_{0}^{\infty}(\mathbf{R})$. If $\varphi \in K$, let $\varphi_{+}$be the function on $\mathbf{R}_{+}$which is equal to $\varphi$ on $\mathbf{R}_{+} \backslash\{0\}$ and is continuous on $\mathbf{R}_{+}$. Then $\varphi_{+}$can be extended to a function $\widetilde{\varphi}_{+} \in \mathcal{D}(\mathbf{R})$; hence $\varphi_{+} \in \mathcal{D}\left(\mathbf{R}_{+}\right)$. Defining $\mathcal{D}\left(\mathbf{R}_{-}\right)$and $\varphi_{-}$similarly, we see that the map

$$
K \ni \varphi \mapsto\left(\varphi_{-}, \varphi_{+}\right) \in \mathcal{D}\left(\mathbf{R}_{-}\right) \times \mathcal{D}\left(\mathbf{R}_{+}\right)
$$


is a bijection which preserves convergence of sequences.

The set of distributions $K^{\prime}$ is defined in the standard way (see $[\mathrm{H}]$ ):

Definition 2.3. A distribution $f$ in $K^{\prime}$ is a linear form on $K$ which is continuous in the sense that $f\left(\varphi_{n}\right) \rightarrow f(\varphi)$ whenever $\varphi_{n}$ tends to $\varphi$ in $K$. A sequence $f_{n} \in K^{\prime}$ is said to converge to $f \in K^{\prime}$ as $n \rightarrow \infty$, if $f_{n}(\varphi) \rightarrow f(\varphi)$ as $n \rightarrow \infty$ for every $\varphi \in K$.

Denote the space of continuous linear forms on $\mathcal{D}\left(\mathbf{R}_{+}\right)$by $\mathcal{D}^{\prime}\left(\mathbf{R}_{+}\right)$. Let $\mathcal{D}_{\mathbf{R}_{-}}(\mathbf{R})$ denote the space of functions in $\mathcal{D}(\mathbf{R})$ that are supported in $\mathbf{R}_{-}$. Viewing $\mathcal{D}\left(\mathbf{R}_{+}\right)$ as the factor space $\mathcal{D}(\mathbf{R}) / \mathcal{D}_{\mathbf{R}_{-}}(\mathbf{R})$ we see that $\mathcal{D}^{\prime}\left(\mathbf{R}_{+}\right)$can be identified with the set of distributions in $\mathcal{D}^{\prime}(\mathbf{R})$ that annihilate all test functions in $\mathcal{D}_{\mathbf{R}_{-}}(\mathbf{R})$; this is of course the set $\mathcal{D}_{\mathbf{R}_{+}}^{\prime}(\mathbf{R})$ of distributions supported in $\mathbf{R}_{+}$. We introduce the analogous notation with $\mathbf{R}_{+}$replaced by $\mathbf{R}_{-}$and vice versa. The space $K^{\prime}$ of generalized distributions can now be identified with the space $\mathcal{D}^{\prime}\left(\mathbf{R}_{-}\right) \times \mathcal{D}^{\prime}\left(\mathbf{R}_{+}\right)$of pairs $\left(f_{-}, f_{+}\right)$of elements $f_{-} \in \mathcal{D}^{\prime}\left(\mathbf{R}_{-}\right), f_{+} \in D^{\prime}\left(\mathbf{R}_{+}\right)$. Indeed, the identification

$$
\mathcal{D}^{\prime}\left(\mathbf{R}_{-}\right) \times \mathcal{D}^{\prime}\left(\mathbf{R}_{+}\right) \ni\left(f_{-}, f_{+}\right) \mapsto f \in K^{\prime}
$$

is defined by

$$
f(\varphi)=f_{-}\left(\varphi_{-}\right)+f_{+}\left(\varphi_{+}\right), \quad \varphi \in K
$$

Using the above observations, it is easy to verify that the identification (2.2) is a vector space isomorphism preserving convergence of sequences.

Since the differentiation operator $d / d x$ preserves the space $\mathcal{D}_{\mathbf{R}_{-}}(\mathbf{R})$, it operates in a natural way in the factor space $\mathcal{D}(\mathbf{R}) / \mathcal{D}_{\mathbf{R}_{-}}(\mathbf{R}) \sim \mathcal{D}\left(\mathbf{R}_{+}\right)$, and the same remark applies of course to $\mathcal{D}\left(\mathbf{R}_{-}\right)$, hence to the product space $\mathcal{D}\left(\mathbf{R}_{-}\right) \times \mathcal{D}\left(\mathbf{R}_{+}\right) \sim K$. This differentiation operator corresponds to pointwise differentiation of functions in $K$. Later we shall sometimes view the elements of $K$ as distributions in $K^{\prime}$ and apply the distribution derivative to them. To distinguish those two operations we shall denote the pointwise differentiation operator by $d / d x$ and the distribution derivative in $K^{\prime}$ by $D_{x}$. The distribution derivative in the space of Schwartz distributions $\mathcal{D}^{\prime}(\mathbf{R})$ will also be denoted $D_{x}$.

Definition 2.4. The generalized derivative $D_{x} f$ of a distribution $f \in K^{\prime}$ is defined by

$$
\left(D_{x} f\right)(\varphi)=-f\left(\frac{d}{d x} \varphi\right) \text { for all } \varphi \in K .
$$

Using (2.2) and the definition of derivative for standard (Schwartz) distributions, we see immediately that

$$
D_{x} f=\left(D_{x} f_{-}, D_{x} f_{+}\right),
$$

where the derivatives applied to $f_{-}$and $f_{+}$are the distribution derivatives in the sense of standard distribution theory.

To any function $f \in L_{\text {loc }}^{1}$ we can associate the linear form $K \ni \varphi \mapsto \int f \varphi d x$, which is an element of $K^{\prime}$. Thus we obtain an imbedding $j: L_{\mathrm{loc}}^{1} \rightarrow K^{\prime}$, and in particular the space of test functions $K$ becomes imbedded in $K^{\prime}$. Using the identification (2.2), we can represent $j(f)$ as the pair $\left(\chi_{-} f, \chi_{+} f\right)$, where $\chi_{-}$and $\chi_{+}$are the characteristic functions of $\mathbf{R}_{-}$and $\mathbf{R}_{+}$, respectively.

If $f$ is absolutely continuous, the Schwartz distribution derivative and the classical pointwize derivative (defined a.e.) of $f$ coincide and are in $L_{\mathrm{loc}}^{1}$, hence are 
elements of $K^{\prime}$, and can be compared to $D_{x} f$, the derivative of $f$ considered as an element of $K^{\prime}$. It turns out that those derivatives do not coincide unless $f(0)=0$.

Example. The derivative of the constant distribution $c \in K^{\prime}$ is equal to the distribution $(-c \delta, c \delta) \in K^{\prime}$, where $\delta \in \mathcal{D}^{\prime}\left(\mathbf{R}_{ \pm}\right)$denotes the Dirac measure at the origin. In fact, using the identification $c=\left(c \chi_{-}, c \chi_{+}\right)$and formula (2.3) we obtain the result.

On the other hand, if $f$ is absolutely continuous and $f(0)=0$, then $D_{x} f$ (in the sense of $\left.K^{\prime}\right)$ is equal to the pointwize derivative $d f / d x$. Indeed, $f=\left(\chi_{-} f, \chi_{+} f\right)$, and since $\chi_{-} f$ and $\chi_{+} f$ are absolutely continuous, the Schwartz distribution derivatives of those functions coincide with the pointwize derivatives.

Lemma 2.1. Assume $f \in K^{\prime}$ and $D_{x} f=0$. Then $f=0$.

Proof. Let $f=\left(f_{-}, f_{+}\right)$. The assumption that $D_{x} f=0$ in $K^{\prime}$ implies that $D_{x} f_{-}=$ $D_{x} f_{+}=0$ in the sense of standard distribution theory; hence $f_{-}$and $f_{+}$are constant functions. But $f_{-}$and $f_{+}$vanish on half-lines; hence $f_{-}=f_{+}=0$.

Corollary. If $f \in L_{\mathrm{loc}}^{1} \subset K^{\prime}$, there is a unique $F \in K^{\prime}$ such that $D_{x} F=f$.

Proof. The function $F(x)=\int_{0}^{x} f(t) d t$ is absolutely continuous and $F(0)=0$; hence $D_{x} F$ (in the sense of $K^{\prime}$ ) is equal to $d f / d x=F(x)$ (defined a.e.). This proves existence, and the uniqueness follows from the lemma.

2.2. The map $\eta: K^{\prime} \rightarrow \mathcal{D}^{\prime}(\mathbf{R})$. Considering an element $f \in K^{\prime}$ as a linear form on $K$, we can construct an element in $\mathcal{D}^{\prime}(\mathbf{R})$ by restricting the linear form to $\mathcal{D}(\mathbf{R})$, which is a subset of $K$. Denote the map from $K^{\prime}$ to $\mathcal{D}^{\prime}(\mathbf{R})$ obtained in this way by $\eta$. If $f=\left(f_{-}, f_{+}\right)$, it is easy to see that $\eta(f)$ can be written $\eta(f)=f_{-}+f_{+}$. The map $\eta$ is obviously not injective, because the element $(h,-h) \in K^{\prime}$ is mapped to zero by $\eta$ for any element $h \in \mathcal{D}^{\prime}(\mathbf{R})$ supported at the origin. That $\eta$ is surjective onto $\mathcal{D}^{\prime}(\mathbf{R})$ can be seen as follows. Let $g$ be arbitrary in $\mathcal{D}^{\prime}(\mathbf{R})$. Write $g=g_{0}+g_{1}$, where $g_{0}$ has compact support and $g_{1}$ vanishes in some neighborhood of the origin. Then $\eta$ maps $\left(\chi_{-} g_{1}, \chi_{+} g_{1}\right)$ to $g_{1}$, so it remains only to consider $g_{0}$. Choose a continuous function $G$ and an integer $k$ such that $D_{x}^{k} G=g_{0}$, and define $F \in K^{\prime}$ by $F=\left(F_{-}, F_{+}\right)=\left(\chi_{-} G, \chi_{+} G\right)$. Then $\eta(F)=F_{-}+F_{+}=G$ and

$$
\eta\left(D_{x}^{k} F\right)=\eta\left(D_{x}^{k}\left(F_{-}, F_{+}\right)\right)=\eta\left(\left(D_{x}^{k} F_{-}, D_{x}^{k} F_{+}\right)\right)=D_{x}^{k} F_{-}+D_{x}^{k} F_{+}=D_{x}^{k} G=g_{0},
$$

which proves that $g \in \eta\left(K^{\prime}\right)$.

The reflexion operator $\mathbf{I}$ is defined for test functions $\varphi \in K$ by $\mathbf{I} \varphi(x)=\varphi(-x)$ and for distributions in $K^{\prime}$ by

$$
(\mathbf{I} f)(\varphi)=f(\mathbf{I} \varphi), \quad \varphi \in K .
$$

The definition of $\mathbf{I} f$ for Schwartz distributions $f$ is analogous to (2.4). If $\varphi \in K$ is equal to $\left(\varphi_{-}, \varphi_{+}\right) \in \mathcal{D}_{\mathbf{R}_{-}}(\mathbf{R}) \times \mathcal{D}_{\mathbf{R}_{+}}(\mathbf{R})$ under the identification (2.1), then clearly $\mathbf{I} \varphi=\left(\mathbf{I} \varphi_{+}, \mathbf{I} \varphi_{-}\right)$. From this we immediately obtain the similar formula for distributions in $K^{\prime}$ : if $f=\left(f_{-}, f_{+}\right) \in K^{\prime}$, then

$$
\mathbf{I} f=\left(\mathbf{I} f_{+}, \mathbf{I} f_{-}\right) \text {. }
$$

This shows that $\eta$ commutes with $\mathbf{I}$ :

$$
\eta(\mathbf{I} f)=\eta\left(\left(\mathbf{I} f_{+}, \mathbf{I} f_{-}\right)\right)=\mathbf{I} f_{+}+\mathbf{I} f_{-}=\mathbf{I}\left(f_{-}+f_{+}\right)=\mathbf{I}(\eta(f)), \quad f \in K^{\prime} .
$$

We say that a distribution $f \in K^{\prime}$ is even if $\mathbf{I} f=f$, and that $f$ is odd if $\mathbf{I} f=-f$. 
Examples. The distributions $(\delta, \delta)$ and $\left(\delta^{\prime},-\delta^{\prime}\right)$ are even, and the distributions $(\delta,-\delta)$ and $\left(\delta^{\prime}, \delta^{\prime}\right)$ are odd. Here $\delta^{\prime}$ denotes the first derivative of the Dirac measure in the space of Schwartz distributions.

The scaling transformation $\mathbf{S}_{c}, c>0$, is defined for test functions $\varphi \in K$ in the usual way:

$$
\mathbf{S}_{c} \varphi(x)=\varphi(c x)
$$

and for distributions $f \in K^{\prime}$ by

$$
\mathbf{S}_{c} f(\varphi)=\frac{1}{c} f\left(\mathbf{S}_{1 / c} \varphi\right), \quad \varphi \in K, \quad c>0 .
$$

Since $\mathbf{S}_{c} \varphi=\left(\mathbf{S}_{c} \varphi_{-}, \mathbf{S}_{c} \varphi_{+}\right)$if $\varphi=\left(\varphi_{-}, \varphi_{+}\right)$, it is easily seen that

$$
\mathbf{S}_{c} f=\left(\mathbf{S}_{c} f_{-}, \mathbf{S}_{c} f_{+}\right), \quad f=\left(f_{-}, f_{+}\right) \in K^{\prime},
$$

where $\mathbf{S}_{c}$ operates on Schwartz distributions in analogy with (2.6). We shall say that $f \in K^{\prime}$ is (positively) homogeneous of order $r \in \mathbf{R}$, if $\mathbf{S}_{c} f=c^{r} f$ for all $c>0$. It is clear then that $f=\left(f_{-}, f_{+}\right) \in K^{\prime}$ is homogeneous of degree $r$ if and only if $f_{-}$and $f_{+}$are homogeneous distributions of degree $r$ in the usual sense. Since $(\delta, \delta) \in K^{\prime}$ is homogeneous of degree -1 , we conclude that its $n$-th derivative (in $\left.K^{\prime}\right)$ is homogeneous of degree $-n-1$.

The map $\eta$ is not invertible. It commutes with the reflection and scaling transformations. If a distribution $g \in D^{\prime}(\mathbf{R})$ has special properties with respect to the reflection and scaling transformations, then there exists a unique distribution $f \in K^{\prime}$ possessing the same properties such that $\eta f=g$.

The following lemma will be needed below.

Lemma 2.2. Assume that the distribution $f \in K^{\prime}$ satisfies the following conditions: $\eta f=D_{x}^{n} \delta$ for some $n \geq 0$ (here $D_{x}^{n} \delta \in \mathcal{D}^{\prime}(\mathbf{R})$ ); $f$ is a homogeneous distribution; and $f$ is an even distribution if $n$ is an even number and an odd distribution if $n$ is odd.

Then $f=D_{x}^{n} \delta$ as a distribution in $K^{\prime}$.

Proof. The first condition implies that $f(\varphi)=0$ for all $\varphi \in C_{0}^{\infty}(\mathbf{R} \backslash\{0\})$; hence $f$ must be supported at the origin. Then $f$ must be a linear combination of derivatives of $\delta$ and $\beta$, and since $f$ is also homogeneous, we know that $f=a D_{x}^{m} \delta+b D_{x}^{m} \beta$ for suitable constants $a, b \in \mathbf{C}$ and $m \in \mathbf{N}$. But $\left(D_{x}^{m} \beta\right)(\varphi)=0$ for all $\varphi \in \mathcal{D}(\mathbf{R})$, whereas $\left(D_{x}^{m} \delta\right)(\varphi)=(-1)^{m} \varphi^{(m)}(0)$ for such $\varphi$; hence $a=1$ and $m=n$. The distribution $D_{x}^{n} \delta \in K^{\prime}$ is even if $n$ is even and odd if $n$ is odd, and $f$ has the same property by assumption, while $\beta$ has the opposite parity; this implies that $b=0$, which completes the proof.

2.3. The classical and generalized derivatives. We introduce two elements in $K^{\prime}$ by

$$
\begin{aligned}
& \delta(\psi)=(\psi(-0)+\psi(+0)) / 2, \\
& \beta(\psi)=(-\psi(-0)+\psi(+0)) / 2,
\end{aligned}
$$

or

$$
\delta=(\delta / 2, \delta / 2), \quad \beta=(-\delta / 2, \delta / 2) .
$$

Every distribution $f \in K^{\prime}$ with support at the origin is equal to a linear combination of the distributions $\delta$ and $\beta$ and their derivatives. The $K^{\prime}$ derivative of $\delta$ will be 
denoted by $\delta^{\prime}$, and $\beta^{\prime}$ will have the analogous meaning. Denote by 1 the function with constant value 1 and by sg the function $x /|x|$. Considering those functions as elements of $K^{\prime}$, we can compute their derivatives:

$$
\begin{gathered}
D_{x} 1=D_{x}\left(\chi_{-}, \chi_{+}\right)=(-\delta, \delta)=2 \beta, \\
D_{x} \mathrm{sg}=D_{x}\left(-\chi_{-}, \chi_{+}\right)=(\delta, \delta)=2 \delta .
\end{gathered}
$$

For arbitrary $\psi \in K$ we now look for constants $A$ and $B$ such that

$$
\psi=A 1+B \mathrm{sg}+\psi_{1},
$$

where $\psi_{1} \in K$ satisfies $\psi_{1}(-0)=\psi_{1}(+0)=0$. It is clear that we must take

$$
\begin{aligned}
& A=(\psi(-0)+\psi(+0)) / 2=\delta(\psi), \\
& B=(-\psi(-0)+\psi(+0)) / 2=\beta(\psi) .
\end{aligned}
$$

Since $\psi_{1}$ is continuous at the origin we have $D_{x} \psi_{1}=(d / d x) \psi$; hence we obtain, by differentiating (2.9) in the sense of $K^{\prime}$,

$$
\begin{aligned}
D_{x} \psi=D_{x} \psi_{1}+A D_{x} 1+B D_{x} \mathrm{sg} & =\frac{d}{d x} \psi_{1}+\delta(\psi) \cdot 2 \beta+\beta(\psi) \cdot 2 \delta \\
& =\frac{d}{d x} \psi+\delta(\psi) \cdot 2 \beta+\beta(\psi) \cdot 2 \delta .
\end{aligned}
$$

Iterating this formula, we obtain

$$
D_{x}^{2} \psi=\left(\frac{d}{d x}\right)^{2} \psi+2 \delta\left(\frac{d \psi}{d x}\right) \beta+2 \beta\left(\frac{d \psi}{d x}\right) \delta+2 \delta(\psi) \beta^{\prime}+2 \beta(\psi) \delta^{\prime} .
$$

Continuing in this way, we finally obtain

$$
D_{x}^{n} \psi=\left(\frac{d}{d x}\right)^{n} \psi+2 \sum_{j=0}^{n-1}(-1)^{j}\left(\delta^{(j)}(\psi) \beta^{(n-j-1)}+\beta^{(j)}(\psi) \delta^{(n-j-1)}\right) .
$$

2.4. Multiplication by test functions. Let $K_{\text {loc }} \subset L_{\text {loc }}^{1}$ denote the set of functions that are $C^{\infty}$ outside the origin and are equal to some element of $K$ in a neighborhood of the origin. Considering the elements of $K_{\text {loc }}$ as distributions in $K^{\prime}$, we want to define the product of $f \in K^{\prime}$ and $\psi \in K_{\text {loc }}$.

Definition 2.5. The product of $f \in K^{\prime}$ and $\psi \in K_{\text {loc }}$ is the element $f \psi \in K^{\prime}$ defined as

$$
(f \psi)(\varphi)=f(\psi \varphi), \quad \varphi \in K
$$

It follows immediately from the definition that $f \psi=\left(f_{-} \psi_{-}, f_{+} \psi_{+}\right)$; here $\psi_{ \pm}=$ $\chi_{ \pm} \psi$. Note that $f_{+} \in \mathcal{D}_{\mathbf{R}_{+}}^{\prime}(\mathbf{R})$, and $\psi_{+} \in \mathcal{D}\left(\mathbf{R}_{+}\right) \sim \mathcal{D}(\mathbf{R}) / \mathcal{D}_{\mathbf{R}_{-}}(\mathbf{R})$. Therefore the product of $f_{+}$and $\psi_{+}$is well defined as a Schwartz distribution, and the result is an element of $\mathcal{D}_{\mathbf{R}_{+}}^{\prime}(\mathbf{R})$.

As an example let us compute the product $\delta \psi$, where $\delta=(\delta / 2, \delta / 2) \in K^{\prime}$ and $\psi \in K_{\text {loc }}$. It is clear that $\delta \psi=0$ if $\psi(-0)=\psi(+0)=0$. Using the representation (2.9) and observing that $\delta \cdot 1=\delta$ and $\delta \cdot \operatorname{sg}=\beta$, we therefore obtain

$$
\delta \psi=A \delta+B \beta=\delta(\psi) \delta+\beta(\psi) \beta .
$$

In the same way we note that $\beta \cdot 1=\beta$ and $\beta \cdot \mathrm{sg}=\delta$, and hence

$$
\beta \psi=A \beta+B \delta=\delta(\psi) \beta+\beta(\psi) \delta .
$$




\section{Finite RANK PERTURBAtions of DifFERENTIAL OPERATORS}

3.1. Singular interactions. Let $L_{0}$ be the selfadjoint operator $\left(i D_{x}\right)^{n}$ with domain equal to $W_{2}^{n}(\mathbf{R})$. We are going to consider additive perturbations of $L_{0}$ corresponding to the formal expression $L=L_{0}+V$, where $V$ is an operator from $W_{2}^{n}(\mathbf{R})$ into $W_{2}^{-n}(\mathbf{R})$. Such an operator is said to be supported at the origin if its Schwartz distribution kernel is supported at the origin in $\mathbf{R} \times \mathbf{R}$, or, which is the same, supp $V f \subset\{0\}$ for all $f \in \operatorname{Dom}(V)$, and $V f=0$ whenever $0 \notin \operatorname{supp} f$. We shall confine ourselves to operators $V$ that act continuously from $W_{2}^{n}(\mathbf{R})$ into $W_{2}^{-n}(\mathbf{R})$, or, equivalently, that satisfy the estimate

$$
|(V f)(\varphi)| \leq C\|f\|_{W_{2}^{n}}\|\varphi\|_{W_{2}^{n}}
$$

for all $f, \varphi \in W_{2}^{n}(\mathbf{R})$. The Schwartz kernel of $V$ must consist of a linear combination of derivatives $D^{k} \delta \otimes D^{l} \delta$ at $(0,0) \in \mathbf{R}^{2}$. Because of (3.1) the possible values of $k$ and $l$ are $0 \leq k \leq n-1,0 \leq l \leq n-1$. Thus $V$ must have the form

$$
V \psi=\sum_{l, m=0}^{n-1} c_{l m} \delta^{(m)}(\psi) \delta^{(l)} .
$$

We also want $V$ to be symmetric in the sense that $(V \psi)(\bar{\varphi})=\overline{(V \varphi)(\bar{\psi})}$, that is, the sesquilinear form $V[\psi, \varphi]=(V \psi)(\bar{\varphi})$ is symmetric. This means that the matrix $\left\{c_{l m}\right\}$ must be Hermitian. Thus our perturbed operator will have the form

$$
L=\left(i D_{x}\right)^{n}+\sum_{l, m=0}^{n-1} c_{l m} \delta^{(m)}(\cdot) \delta^{(l)},
$$

where $\left\{c_{l m}\right\}$ is an Hermitian matrix.

The linear operator defined by the formal differential expression (3.2) restricted to $C_{0}^{\infty}(\mathbf{R} \backslash\{0\})$ coincides with the operator $L_{0}^{0}=\left.L_{0}\right|_{C_{0}^{\infty}(\mathbf{R} \backslash\{0\})}$. It follows that any selfadjoint operator corresponding to the formal expression (3.2) must coincide with some selfadjoint extension of $L_{0}^{0}$.

3.2. Selfadjoint extensions of $L_{0}^{0}$. We study in this section the family of selfadjoint extensions of the symmetric operator $L_{0}^{0}$. The domain of the adjoint operator $L_{0}^{0 *}$ is equal to $W_{2}^{n}(\mathbf{R} \backslash\{0\})$. The deficiency indices of the operator $L_{0}^{0}$ are equal to $(n, n)$. For given $z$ with $\Im z \neq 0$ the deficiency subspace $N_{z}$ consists of the set of solutions $g \in W_{2}^{n}(\mathbf{R} \backslash\{0\})$ of the equation

$$
\left(i \frac{d}{d x}\right)^{n} g(x)=z g(x) .
$$

Any element of $N_{z}$ can be written as a linear combination of functions of the form

$$
g(x)=\Theta(-\Im \lambda x) \exp (-i \lambda x)
$$

where $\lambda$ is some complex root of the equation $\lambda^{n}=z$.

For $f \in W_{2}^{n}(\mathbf{R} \backslash\{0\})$ let $J_{n}^{+} f$ be the vector

$$
J_{n}^{+} f=\left(f(+0), f^{\prime}(+0), \ldots, f^{(n-1)}(+0)\right) \in \mathbf{C}^{n},
$$

let $J_{n}^{-} f$ be defined similarly, and set furthermore

$$
J_{n} f=\left(J_{n}^{-} f, J_{n}^{+} f\right) \in \mathbf{C}^{2 n} .
$$

Lemma 3.1. For any $z$ with $\Im z \neq 0$ the map $J_{n}$ is a bijection from $N_{z} \oplus N_{\bar{z}}$ onto $\mathbf{C}^{2 n}$. 
Proof. Since $J_{n}$ is a mapping between spaces of the same dimension, it is enough to prove that $J_{n}$ is injective. Thus assume that $u \in N_{z}, v \in N_{\bar{z}}$, and $J_{n}(u+v)=0$. Then

$$
\int_{0}^{\infty}\left(i \frac{d}{d x}\right)^{n}(u(x)+v(x)) \overline{u(x)} d x=\int_{0}^{\infty}(u(x)+v(x)) \overline{\left(i D_{x}\right)^{n} u(x)} d x,
$$

since the boundary terms vanish by assumption. Using (3.3), we obtain

or

$$
\int_{0}^{\infty}(z u(x)+\bar{z} v(x)) \overline{u(x)} d x=\int_{0}^{\infty}(u(x)+v(x)) \overline{z u(x)} d x
$$

$$
(z-\bar{z}) \int_{0}^{\infty}|u(x)|^{2} d x=0 .
$$

Since $\Im z \neq 0$, it follows that $u(x)=0$ for $x>0$. In the same way we can prove that $u$ and $v$ both vanish on all of $\mathbf{R}$.

Let us introduce the $2 n$-dimensional vectors

$$
\begin{aligned}
& \vec{\delta}(f)=I\left(J_{n}^{+} f+J_{n}^{-} f\right) / 2, \\
& \vec{\beta}(f)=I\left(J_{n}^{+} f-J_{n}^{-} f\right) / 2,
\end{aligned}
$$

where $I$ is the $n \times n$ diagonal matrix with diagonal entries $I_{l l}=(-1)^{l}, l=0,1, \ldots$, $n-1$. It follows that

$$
J_{n}^{ \pm} f=I(\vec{\delta}(f) \pm \vec{\beta}(f)) .
$$

Lemma 3.2. The boundary form of the adjoint operator $L_{0}^{0 *}$ is equal to

$$
\begin{gathered}
\left\langle L_{0}^{0 *} u, v\right\rangle-\left\langle u, L_{0}^{0 *} v\right\rangle=i^{n}\left\{-\left\langle I A J_{n}^{+} u, J_{n}^{+} v\right\rangle_{\mathbf{C}^{n}}+\left\langle I A J_{n}^{-} u, J_{n}^{-} v\right\rangle_{\mathbf{C}^{n}}\right\} \\
=2(-i)^{n}\left\{\langle I A \vec{\delta}(u), \vec{\beta}(v)\rangle_{\mathbf{C}^{n}}+\langle I A \vec{\beta}(u), \vec{\delta}(v)\rangle_{\mathbf{C}^{n}}\right\},
\end{gathered}
$$

where $A$ is the $n \times n$ antidiagonal matrix with the coefficients

$$
A_{l m}=\delta_{l+m}^{n-1}, \quad l, m=0,1, \ldots, n-1 .
$$

Proof. The first equality in (3.6) is obtained by partial integrations. Inserting the expressions (3.5) for $J_{n}^{ \pm}$gives

$$
\left\langle L_{0}^{0 *} u, v\right\rangle-\left\langle u, L_{0}^{0 *} v\right\rangle=-2 i^{n}\left\{\langle A I \vec{\delta}(u), \vec{\beta}(v)\rangle_{\mathbf{C}^{n}}+\langle A I \vec{\beta}(u), \vec{\delta}(v)\rangle_{\mathbf{C}^{n}}\right\} .
$$

The second part of (3.6) now follows from the matrix identity $A I=(-1)^{n-1} I A$.

To every selfadjoint extension $L$ of $L_{0}^{0}$ we can associate a subspace $E \subset \mathbf{C}^{2 n}$

$$
E=J_{n}(\operatorname{Dom}(L))
$$

consisting of all boundary values of elements in the domain of $L$.

Using the expression (3.6) for the boundary form of $L_{0}^{0 *}$, we can define a sesquilinear form on $\mathbf{C}^{2 n}$ as follows. For $s=\left(s_{-}, s_{+}\right)$and $t=\left(t_{-}, t_{+}\right) \in \mathbf{C}^{2 n}$ we set

$$
\mathbf{Q}[s, t]=\left\langle I A s_{+}, t_{+}\right\rangle_{\mathbf{C}^{n}}-\left\langle I A s_{-}, t_{-}\right\rangle_{\mathbf{C}^{n}} .
$$

Then (3.6) can be written

$$
\left\langle L_{0}^{0 *} u, v\right\rangle-\left\langle u, L_{0}^{0 *} v\right\rangle=-i^{n} \mathbf{Q}\left[J_{n} u, J_{n} v\right] .
$$

The form $\mathbf{Q}$ is skew-Hermitian and nondegenerate. Moreover $\overline{\mathbf{Q}[s, t]}=\mathbf{Q}[\bar{s}, \bar{t}]$ for all $s, t \in \mathbf{C}^{2 n}$; hence the corresponding bilinear form $\mathbf{W}[s, t]=\mathbf{Q}[s, \bar{t}]$ is symplectic. 
Therefore the form $\mathbf{Q}$ can be called sesquilinear symplectic. Every vector space with a sesquilinear symplectic structure has Lagrangian planes, i.e. subspaces $E$ which are orthogonal to themselves with respect to the form $\mathbf{Q}: E=E^{\perp}$. All Lagrangian planes have dimension $n$, since $\mathbf{Q}$ is nondegenerate, which implies $\operatorname{dim} E+\operatorname{dim} E^{\perp}=$ $2 n$.

Theorem 3.1. The map $\operatorname{Dom}(L) \mapsto J_{n}(\operatorname{Dom}(L)) \subset \mathbf{C}^{2 n}$ defines a 1-1 correspondence between the set of selfadjoint extensions of $L_{0}^{0}$ and the set of subspaces of $\mathbf{C}^{2 n}$ that are Lagrangian with respect to the form $\mathbf{Q}$.

Proof. Given a selfadjoint extension $L$, it follows from Lemma 3.2 that the form $\mathbf{Q}$ vanishes on the subspace (3.7) of $\mathbf{C}^{2 n}$. If the dimension of $E$ were less than $n$, then the elements of the domain of $L$ would span a subspace of $N_{z} \oplus N_{\bar{z}}$ with dimension less than $n$, and this would imply that $L$ is not selfadjoint. Therefore $E$ must be $n$-dimensional and hence Lagrangian.

Conversely, let $E$ be Lagrangian. Let the domain of $L$ be defined by (3.7). Then the operator $L$ is symmetric. Selfadjointness of $L$ follows from the fact that the map $J_{n}$ restricted to $N_{z} \oplus N_{\bar{z}}$ is injective (Lemma 3.1).

3.3. Singular interactions and selfadjoint perturbations. We shall now apply the distribution theory developed in Section 2 to define a domain for the operator $L$ in (3.2). If the Dirac distributions in (3.2) are interpreted as elements of $K^{\prime}$ and $W_{2}^{n}(\mathbf{R} \backslash\{0\})$ is considered as a subset of $K^{\prime}$, then the operator $L$ maps $W_{2}^{n}(\mathbf{R} \backslash\{0\})$ into $K^{\prime}$. Note that the differential operator $D_{x}$ then has to be understood according to Definition 2.4. Composing with the map $\eta: K^{\prime} \rightarrow \mathcal{D}^{\prime}(\mathbf{R})$, we get an operator $u \mapsto \eta(L u)$ from $W_{2}^{n}(\mathbf{R} \backslash\{0\})$ into $\mathcal{D}^{\prime}(\mathbf{R})$. We can then define a new operator $\mathbf{L}$ in $L_{2}(\mathbf{R})$ as $\mathbf{L} u=\eta(L u)$ with the domain

$$
\operatorname{Dom}(\mathbf{L})=\left\{u \in W_{2}^{n}(\mathbf{R} \backslash\{0\}) ; \mathbf{L} u \in L_{2}(\mathbf{R})\right\} .
$$

Theorem 3.2. The operator

$$
\mathbf{L}=\eta\left(\left(i D_{x}\right)^{n}+\sum_{l, m=0}^{n-1} c_{l m} \delta^{(m)}(\cdot) \delta^{(l)}\right)
$$

defined by the Hermitian matrix $C=\left\{c_{l m}\right\}_{l, m=0}^{n-1}$ is selfadjoint on the domain (3.8). The operator $\mathbf{L}$ coincides with the operator $L_{0}^{0 *}$ restricted to the domain of functions $u \in W_{2}^{n}(\mathbf{R} \backslash\{0\})$ satisfying the following boundary condition at the origin:

$$
2 \vec{\beta}(u)=-(-i)^{n} I A C \vec{\delta}(u) .
$$

Proof. If $L$ acts from $W_{2}^{n}(\mathbf{R} \backslash\{0\}) \subset K^{\prime}$ into $K^{\prime}$ as explained above, then by (2.10)

$$
\begin{aligned}
L u=i^{n} v & +2 i^{n} \sum_{l=0}^{n-1}(-1)^{n-l-1}\left(\delta^{(n-l-1)}(u) \beta^{(l)}+\beta^{(n-l-1)}(u) \delta^{(l)}\right) \\
& +\sum_{l, m=0}^{n-1} c_{l m} \delta^{(m)}(u) \delta^{(l)},
\end{aligned}
$$

where $v=(d / d x)^{n} u \in L_{2}(\mathbf{R})$. Note that here $\delta^{(j)} \in K^{\prime}$, and hence

$$
\delta^{(j)}(u)=\frac{(-1)^{j}}{2}\left(u^{(j)}(+0)+u^{(j)}(-0)\right) .
$$


Since $\eta\left(\beta^{(j)}\right)=0$ and $\eta\left(\delta^{(j)}\right)=\delta^{(j)} \in \mathcal{D}^{\prime}(\mathbf{R})$, this gives

$$
\mathbf{L} u=i^{n} v+2 i^{n} \sum_{l=0}^{n-1}(-1)^{n-l-1} \beta^{(n-l-1)}(u) \delta^{(l)}+\sum_{l, m=0}^{n-1} c_{l m} \delta^{(m)}(u) \delta^{(l)} .
$$

The requirement that $\mathbf{L} u \in L_{2}(\mathbf{R})$ then means that for each $l$

$$
2 i^{n}(-1)^{n-l-1} \beta^{(n-l-1)}(u)+\sum_{m=0}^{n-1} c_{l m} \delta^{(m)}(u)=0
$$

which is the formula (3.9). This boundary condition defines a certain Lagrangian plane in $\mathbf{C}^{2 n}$, and it follows from Theorem 3.1 that the restricted operator is selfadjoint.

3.4. Singular interactions with infinite coupling. The operators with singular interaction form a proper subset of the set of all selfadjoint perturbations of the operator $L_{0}$. This is related to the fact that not every Langrangean subspace of $\mathbf{C}^{2 n}$ can be described by a relation of the form (3.9). Let $C$ be presented in the form $C=C_{0}^{-1} C_{1}$, where $C_{0}, C_{1}$ are Hermitian $n \times n$ matrices. Then the boundary conditions (3.9) can be written as

$$
2 C_{0} I A \vec{\beta}(\psi)=i^{n} C_{1} \vec{\delta}(\psi) .
$$

The last equation can be considered even if the matrix $C_{0}$ is not invertible. This situation corresponds formally to the matrices $C$ with infinite determinant, and can be considered as singular interaction with infinite coupling. The case of rank one perturbations with infinite coupling was considered in [GeSi]. Singular interactions with infinite coupling for second order differential operators were studied in [Ku]. Every Lagrangian subspace of $\mathbf{C}^{2 n}$ can be described by conditions of the type (3.12). Thus the set of selfadjoint extensions of the operator $L_{0}^{0}$ coincides with the set of singular interactions with finite or infinite coupling.

\section{ACKNOWLEDGMENT}

The first author thanks the Alexander von Humboldt Foundation for financial support.

\section{REFERENCES}

[AGHKH] S. Albeverio, F. Gesztesy, R. Hoegh-Krohn, H. Holden, "Solvable Models in Quantum Mechanics", Springer-Verlag, 1988. MR 90a:81021

[AlKu] S. Albeverio, P. Kurasov, Rank one perturbations, approximations and selfadjoint extensions, J. Func. Anal. 148 (1997), 152-169.

[AlKu2] S. Albeverio, P. Kurasov, Rank one perturbations of not semibounded operators, Itegr. Eq. Oper. Theory 27 (1997), 379-400.

[AlKu3] S. Albeverio, P. Kurasov, Finite rank perturbations and distributions theory, SFB 237 - Preprint No. 368, Bochum, Germany (1997).

[BeFad] F. Berezin, L. Faddeev, A remark on Schrödinger's equation with a singular potential, Dokl. Akad. Nauk. SSSR 137, N5 (1961), 1011-1014. MR 23:B2345

[GeSi] F. Gesztesy, B. Simon, Rank one perturbations at infinite coupling, J. Func. Anal. 128 (1995), 245-252. MR 95m:47014

[H] L. Hörmander, "The Analysis of Linear Partial Differential Operators I", SpringerVerlag, Berlin, 1983. MR 85g:35002a

[KiSi] A. Kiselev, B. Simon, Rank one perturbations with infinitesimal coupling, J. Func. Anal. 130 (1995), 345-356. MR 96e:47012 
$[\mathrm{Ku}] \quad$ P. Kurasov, Distribution theory for discontinuous test functions and differential operators with generalized coefficients, J. Math. Anal. Appl. 201 (1996), 297-323. CMP 96:15

[ReSi] M. Reed, B. Simon, "Methods of modern mathematical physics", Vol. II, Academic Press, New York, 1975. MR 58:12429b

[Si] B. Simon, Spectral analysis of rank one perturbations and applications, CRM Proceedings and Lectures Notes Vol. 8 (1995), 109-149. MR 97c:47008

(P. Kurasov) Department of Mathematics, Stockholm University, S-10691 Stockholm, Sweden; Department of Mathematics, Lule̊ University, S-97187 Luleå, Sweden; Department of Mathematical Physics, St.Petersburg University, 198904 St.Petersburg, Russia

E-mail address: pak@matematik.su.se

(J.Boman) Department of Mathematics, Stockholm University, S-10691 Stockholm, SWEDEN

E-mail address: jabo@matematik.su.se 